\title{
Le silicium polycristallin Polix : élaboration, propriétés et performances
}

\author{
G. Nouet, P. Lay (*) et J. L. Chermant \\ Laboratoire de Cristallographie, Chimie et Physique des Solides, U.A. 251 CNRS-ISMRa, Université, \\ 14032 Caen Cedex, France
}

(Reçu le 28 novembre 1986, accepté le 27 janvier 1987)

\begin{abstract}
Résumé. - Les lingots de silicium polycristallin sont préparés par solidification unidirectionnelle, l'originalité de la méthode est due à l'utilisation d'un encapsulant qui agit comme une barrière entre le creuset et le bain liquide. L'analyse de la morphologie et des caractéristiques structurales, ainsi que l'analyse chimique ont permis de suivre l'influence des différents paramètres sur les propriétés photovoltaïques des cellules solaires. Leur optimisation a donné des rendements photovoltaïques de $11 \%$. Les conditions d'utilisation de silicium de qualité moindre sont définies.
\end{abstract}

\begin{abstract}
Polycrystalline silicon ingots are grown by unidirectional solidification. An encapsulant is used to prevent impurities of the crucible from diffusing into the liquid bath. The influence of thermal, physical parameters on the photovoltaic properties of solar cells are controlled by morphological, structural, chemical analysis. Photovoltaic efficiency equal to $11 \%$ is obtained after optimizing. Lower-grade silicon can be used provided that some specifications are respected.
\end{abstract}

\section{Introduction.}

Le choix du développement de la filière multicristalline s'inscrit dans le programme de production d'énergie photovoltaïque à faible coût. Après une période de mise au point et d'automatisation du procédé de réalisation des cellules, la part du matériau est devenue prépondérante dans le coût final des modules, elle avait atteint $60 \%$ en 1983 . L'un des axes de réduction du coût a été l'utilisation de silicium multicristallin afin de réduire le prix des plaques d'au moins $50 \%$. Sa faible disponibilité a contraint les sociétés souhaitant survivre à s'impliquer dans la croissance et le sciage des lingots.

La Compagnie Générale d'Electricité à été à l'origine du projet d'élaboration de lingots de silicium multicristallin à solidification orientée appelé POLIX. Le programme de recherche et développement de la méthode a été assuré aux Laboratoires de Marcoussis (LDM-CGE) et à Photowatt Int. (Caen).

(*) Photowatt Int. S.A., 6, rue de la Girafe, BP 5117, 14043 Caen Cedex, France.

REVUE DE PHYSIQUE APPLIQUÉE. - T. 22, N` 7, JUILLET 198
Le principe d'élaboration a été étudié aux LDM de 1979 à 1982 sur un four de laboratoire ayant une capacité de $1 \mathrm{~kg}$ puis sur un four expérimental de $20 \mathrm{~kg}$. Le développement a été assuré à Photowatt Int. avec la conception et la réalisation d'un four pilote ayant une capacité de $60 \mathrm{~kg}$ suivie d'études d'accompagnement. En juin 1983, les lingots de $12 \mathrm{~kg}$ présentaient des rendements moyens de $9 \%$ pour atteindre $55 \mathrm{~kg}$ et $11 \%$ en 1986 .

Le développement de ce programme a montré que la filière Polix est particulièrement attractive à court et moyen terme. A long terme, cette filière ne demeurera compétitive par rapport aux filières concurrentes : silicium amorphe et silicium en ruban qu'à deux conditions :

- une diminution très importante de la quantité de silicium nécessaire pour réaliser un mégawattcrète de cellules solaires. A cet effet un programme de développement d'une méthode de sciage des lingots à l'aide d'une machine à fil a été entrepris et associé à la réduction de l'épaisseur des plaquettes de 400 à $200 \mu \mathrm{m}$, soit une diminution de plus de $50 \%$ de la quantité de matière nécessaire par wattcrète, 
- une disponibilité en grande quantité de silicium-matière première à un prix actuel inférieur à $150 \mathrm{~F} / \mathrm{kg}$. La charge actuellement utilisée est soit de qualité électronique à un prix très élevé, $300 \mathrm{~F} / \mathrm{kg}$, soit des rejets de l'industrie électronique traités mécaniquement ou chimiquement; le problème essentiel est alors le suivi de l'approvisionnement. Le développement d'une méthode de purification du silicium métallurgique pourrait en assurer la régularité. Ce procédé a été étudié à l'E.N.S.C.P. dans le laboratoire du Professeur Amouroux et son développement industriel est envisagé.

\section{Méthode d'élaboration.}

2.1 SOLIDIFICATION ORIENTÉE. - Le principe d'élaboration des lingots de silicium polycristallin est basé sur une méthode de cristallisation orientée dans un creuset fixe soumis à un écoulement de chaleur unidirectionnel.

La fusion de la charge de silicium est réalisée dans un creuset en graphite calorifugé sur toute sa surface. L'ajout d'un agent encapsulant à la charge permet d'éviter la contamination du silicium par le creuset et facilite la récupération du lingot [1]. La température du bain est stabilisée à une température légèrement supérieure à la température de fusion du silicium $\left(1420^{\circ} \mathrm{C}\right)$. En maintenant constante la puissance délivrée par le générateur à la valeur déterminée lors de la phase de stabilisation, la cristallisation est obtenue en supprimant une partie du calorifuge de la paroi inférieure du creuset (Fig. 1). Ceci permet l'évacuation de la chaleur latente de solidification et conduit à une solidification dirigée.

La phase suivante consiste à récupérer le lingot par retournement du creuset en utilisant les proprié-

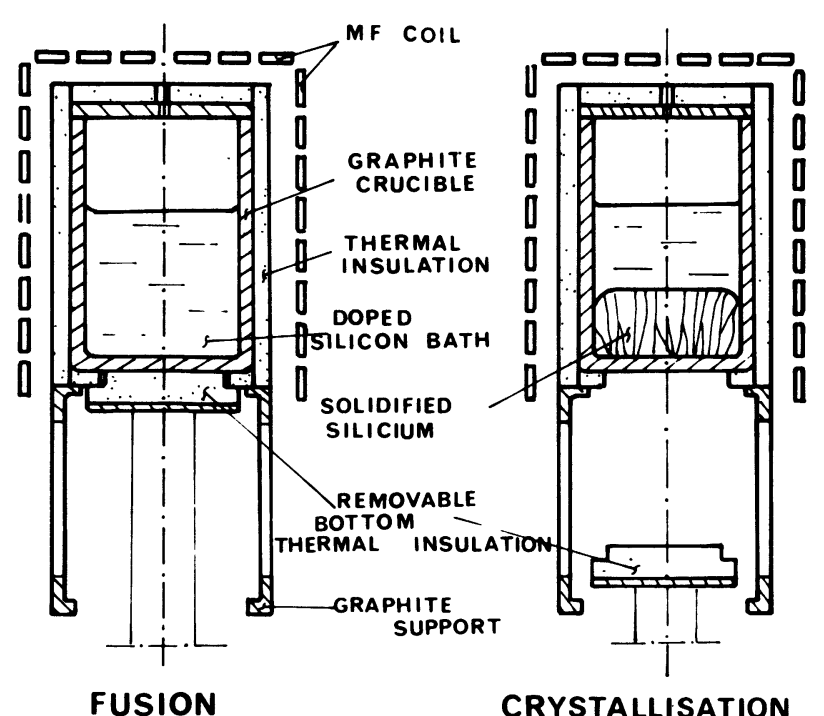

Fig. 1. - Méthode de cristallisation.

[Method of crystallization.] tés de l'agent encapsulant. Le lingot est ensuite refroidi dans un réceptable calorifugé. Cette technique de cristallisation avec creuset fixe simplifie la technologie et favorise la planéité du front de solidification.

La méthode de récupération du lingot permet d'une part de réutiliser le creuset et d'autre part de le refroidir à l'extérieur, et de s'affranchir des difficultés liées à la différence des coefficients de dilatation du graphite et du silicium au cours du refroidissement. L'encapsulation des lingots est aussi utilisée pour éviter la diffusion des impuretés du creuset vers le silicium.

2.2 Développement De la méthode. - Afin de préparer l'industrialisation du procédé, un four pilote a été conçu et réalisé. L'objectif était d'assurer une productivité satisfaisante en optimisant la durée du cycle d'élaboration tout en maintenant une bonne qualité cristalline.

L'expérience acquise avec l'élaboration de lingots de $15 \mathrm{~kg}$ associée à une simulation thermique de la solidification a permis de préciser l'extrapolation à des lingots de grandes dimensions. Les principaux paramètres de cristallisation sont la hauteur des lingots, la taille de la surface émissive et la température de stabilisation du bain de silicium avant le début de la solidification. Comme la durée de solidification est directement liée à la hauteur du bain de silicium, l'extrapolation à des blocs de grandes dimensions a été réalisée préférentiellement en largeur pour obtenir des lingots de $30 \times 30 \times 12 \mathrm{~cm}^{3}$ ou $40 \times 40 \times 13 \mathrm{~cm}^{3}$ soit respectivement $30 \mathrm{~kg}$ en neuf blocs $10 \times 10 \times 12 \mathrm{~cm}^{3}$ ou $55 \mathrm{~kg}$ en seize blocs de $10 \times 10 \times 13 \mathrm{~cm}^{3}$. Après la solidification, le creuset est retourné afin de récupérer le lingot qui, une fois démoulé, est refroidi suivant un programme thermique. Il est ensuite transféré dans un sas où le refroidissement s'effectue jusqu'à la température ambiante. Cette dernière opération a donc lieu en temps masqué. Les durées d'occupation du four pour l'élaboration d'un lingot de 55 à $60 \mathrm{~kg}$ sont voisines de douze heures [2, 3]. Un cycle complet est présenté dans la figure 2 .

Comme nous le verrons par la suite, les propriétés du matériau résultent des conditions de croissance. En effet, la croissance est gouvernée par deux phénomènes : les transferts thermiques et les transferts de matière auxquels sont directement liés la qualité cristalline et l'état de pureté. Le lieu privilégié de ces transferts est l'interface de solidification.

La démarche suivie au cours du développement du procédé a donc consisté à mettre en évidence les causes de limitation des performances puis à agir sur les conditions de croissance afin d'améliorer la qualité du matériau. Le but étant la production de cellules solaires, des prélèvements ont été effectués 


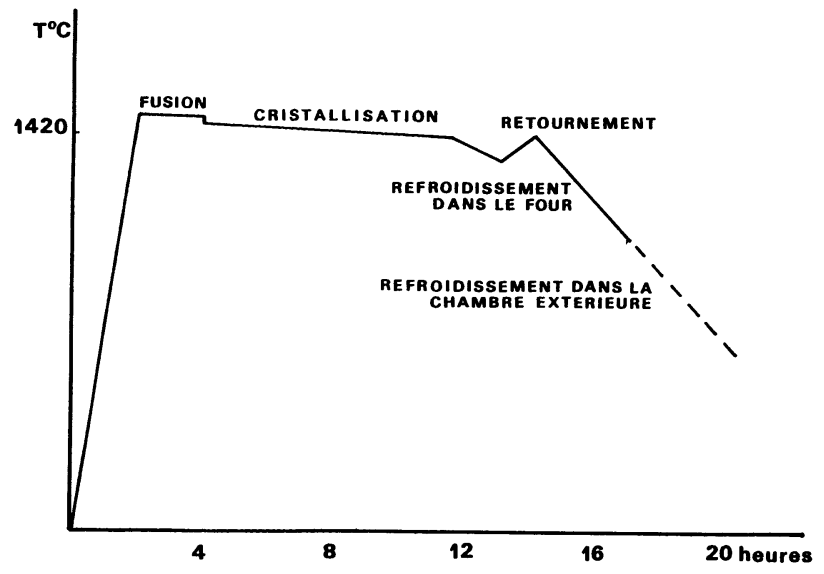

Fig. 2. - Cycle thermique de fusion et solidification.

[Thermal cycle for the melting and the solidification of 30 $60 \mathrm{~kg}$ ingots.]

sur tous les lingots élaborés et les performances photovoltaïques ont été analysées.

\section{Analyse morphologique.}

La solidification unidirectionnelle du lingot s'accompagne évidemment d'une structure morphologique très hétérogène. Même dans le plan normal à la direction de croissance la distribution de la taille des grains ainsi que leur forme peuvent être très variées (Fig. 3). De très grandes hétérogénéités apparentes sont susceptibles de modifier fortement une simple

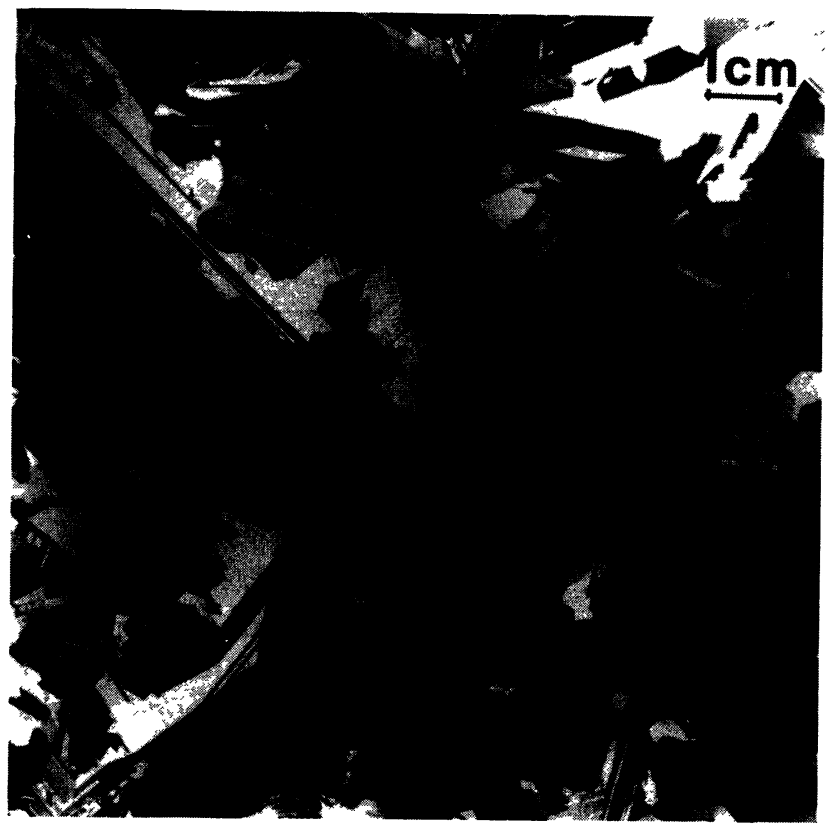

Fig. 3. - Microstructure d'une plaque découpée perpendiculairement à l'axe de croissance.

[Microstructure of a wafer cut normal to the growth axis.] analyse. Notre choix s'est alors porté sur une méthode quantitative par analyse d'images automatique, ayant pour objectif l'obtention d'informations morphologiques sur la croissance : apparition ou élimination de grains au cours de la solidification, détection éventuelle d'une anisotropie.

3.1 MÉTHOde. - Les analyses sont effectuées en utilisant un analyseur de texture Leitz TAS travaillant selon les principes de la morphologie avec un réseau hexagonal.

A partir d'un microscope optique ou d'un épidiascope, l'image à analyser est envoyée sur une caméra TV qui délivre un signal électrique seuillé au gré de l'utilisateur avant d'être digitalisé. Les mesures ou les transformations d'images sont réalisées directement sur cette image digitalisée.

3.2 AnAlyse granulométrique. - Par définition, l'analyse granulométrique consiste à suivre l'évolution d'une grandeur en fonction d'un paramètre décrivant la taille. La fonction $P(l)$ qui est la probabilité d'implanter un segment de longueur $l$ dans la phase a été retenue. Cette granulométrie est possible en nombre et en mesure ; de plus, l'analyse selon plusieurs directions permet de mesurer l'anisotropie éventuelle du matériau selon sa position par rapport aux faces du creuset. Expérimentalement les grandeurs suivantes ainsi que leur distribution sont calculées à partir de cette fonction $P(l)$ :

$L$ : la traversée moyenne en nombre ou la corde moyenne en nombre,

$M$ : la traversée moyenne en mesure ou la corde moyenne en mesure,

$L_{\mathrm{A}}$ : le périmètre spécifique lié directement à la dérivée de la fonction $P(l)$,

$S_{\mathrm{V}}$ : la surface spécifique.

3.2.1 Evolution de la taille des grains. - Pour déterminer l'influence des différents paramètres liés à l'élaboration du lingot susceptibles de modifier la granulométrie, plusieurs plaques issues d'un même lingot sont testées. Ainsi les influences des parois du creuset, des dimensions du lingot, de la surface émissive ont été testées. Les valeurs des différentes grandeurs sont rassemblées dans les tableaux I et II.

Le lingot de $13 \mathrm{~kg}$ a été solidifié par dégagement total de la surface émissive et sans palier thermique ultérieur. A l'exception des deux plaques supérieures, les traversées moyennes augmentent régulièrement de bas en haut. Comme la forme moyenne des grains n'évolue pas le long du lingot, on constate qu'il y a élimination régulière de grains pendant la croissance. Cette évolution est due au dégagement total de la surface émissive qui provoque une forte nucléation à la base du lingot. La vitesse de refroidissement diminuant avec la hauteur de silicium solidifié, la taille des grains augmente régulièrement. 
Tableau I. - Evolution des grandeurs morphologiques selon l'axe de croissance ( $n^{\mathrm{o}}$ faible : haut du lingot, $n^{\circ}$ élevé : bas du lingot) pour un lingot de $13 \mathrm{~kg}$.

[Morphological parameters along the growth axis (low number : ingot top, high number : ingot bottom) for a $13 \mathrm{~kg}$ ingot.]

\begin{tabular}{|l|c|c|c|c|}
\hline $\mathrm{n}^{\bullet}$ plaque & $\begin{array}{c}\text { Traversée } \\
\text { moyenne en } \\
\text { mesure } \mathrm{mm} .\end{array}$ & $\begin{array}{c}\text { Traversée } \\
\text { moyenne en } \\
\text { nombre } \mathrm{mm} .\end{array}$ & $\begin{array}{c}\text { Périmètre } \\
\text { spéc1fique } \\
\mathrm{mm}^{-1}\end{array}$ & $\begin{array}{c}\text { Surface } \\
\text { opécifique } \\
\mathrm{mm}-1 .\end{array}$ \\
\hline 26 (haut) & 5,6 & 2,8 & 0,96 & 1,22 \\
27 & 5,4 & 2,8 & 0.96 & 1,22 \\
51 & 6,35 & 2,9 & 0,96 & 1,23 \\
52 & 6,5 & 3,0 & 0,95 & 1,21 \\
76 & 4,4 & 2,5 & 1,06 & 1,35 \\
77 & 4,8 & 2,7 & 1,03 & 1,32 \\
101 & 3,9 & 2,2 & 1,25 & 1,59 \\
102 & 4,0 & 2,1 & 1,28 & 1,63 \\
120 & 3,7 & 1,95 & 1,37 & 1,75 \\
121 (bas) & 3,55 & 1,96 & 1,38 & 1,76 \\
\hline
\end{tabular}

Tableau II. - Evolution des mêmes grandeurs pour un lingot de $50 \mathrm{~kg}$.

[The same parameters for a $55 \mathrm{~kg}$ ingot.]

\begin{tabular}{|l|c|c|c|c|}
\hline$n^{\circ}$ plaque & $\begin{array}{l}\text { Traversée } \\
\text { moyenne en } \\
\text { mesure } \mathrm{mm} .\end{array}$ & $\begin{array}{c}\text { Traversée } \\
\text { moyenne en } \\
\text { nombre } \mathrm{mm} .\end{array}$ & $\begin{array}{c}\text { Pér1mètre } \\
\text { spécifique } \\
\mathrm{mm}^{-1}\end{array}$ & $\begin{array}{c}\text { Surface } \\
\text { spécifique } \\
\mathrm{mm}^{-1}\end{array}$ \\
\hline 99 (haut) & 8,0 & 3,6 & 0,79 & 1,01 \\
90 & 7,6 & 3,3 & 0,85 & 1,09 \\
80 & 7,2 & 3,4 & 0,84 & 1,07 \\
70 & 7,0 & 3,3 & 0,86 & 1,09 \\
60 & 7,1 & 3,2 & 0,88 & 1,12 \\
50 & 7,3 & 3,2 & 0,88 & 1,12 \\
35 & 7,1 & 3,15 & 0,90 & 1,14 \\
30 & 7,5 & 3,35 & 0,85 & 1,08 \\
20 & 7,2 & 3,2 & 0,89 & 1,13 \\
10 & 6,9 & 3,2 & 0,89 & 1,13 \\
7 (bas) & 7,5 & 3,5 & 0,82 & 1,04 \\
\hline
\end{tabular}

Pour le lingot de $50 \mathrm{~kg}$, les plaques analysées ont été prélevées dans sa partie centrale. Ce lingot représente un état standard de la production selon le cycle thermique défini précédemment. Les traversées moyennes varient très peu et sont caractéristiques d'une croissance normale et régulière. Ces résultats constituent donc une référence de la morphologie pour les lingots élaborés selon le procédé standard.

3.2.2 Etude de l'anisotropie. - Bien que la fonction $P(l)$ puisse caractériser l'anisotropie d'une structure, son utilisation devient délicate lorsque la structure possède plus d'une orientation préférentielle. Il est alors préférable d'utiliser la rose des directions qui n'est rien d'autre que la longueur des joints de grains par unité de surface ayant une orientation comprise dans un écart angulaire [4].

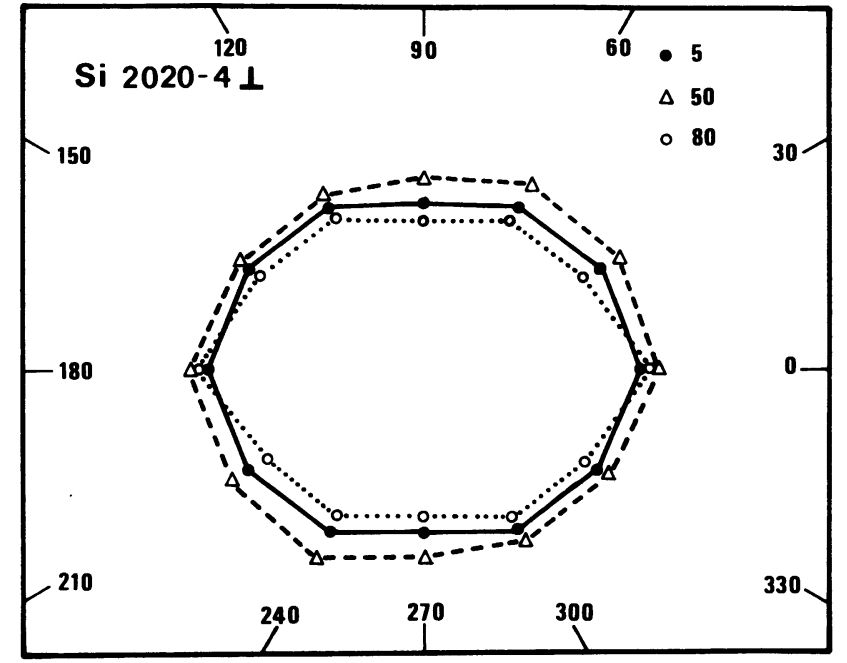

Fig. 4. - Rose des directions montrant une structure parfaitement isotrope dans un bloc d'angle pour un lingot de $55 \mathrm{~kg}$, structure qui est maintenue de la base du lingot (plaque $n^{\circ} 80$ ) à sa partie supérieure (plaque $n^{\circ} 5$ ) [4].

[Rose of directions showing a perfectly isotrop structure in a corner block of a $55 \mathrm{~kg}$ ingot, this structure is retained from the bottom (wafer $n^{\circ} 80$ ) to the top (wafer $n^{\circ} 5$ ) [4].]

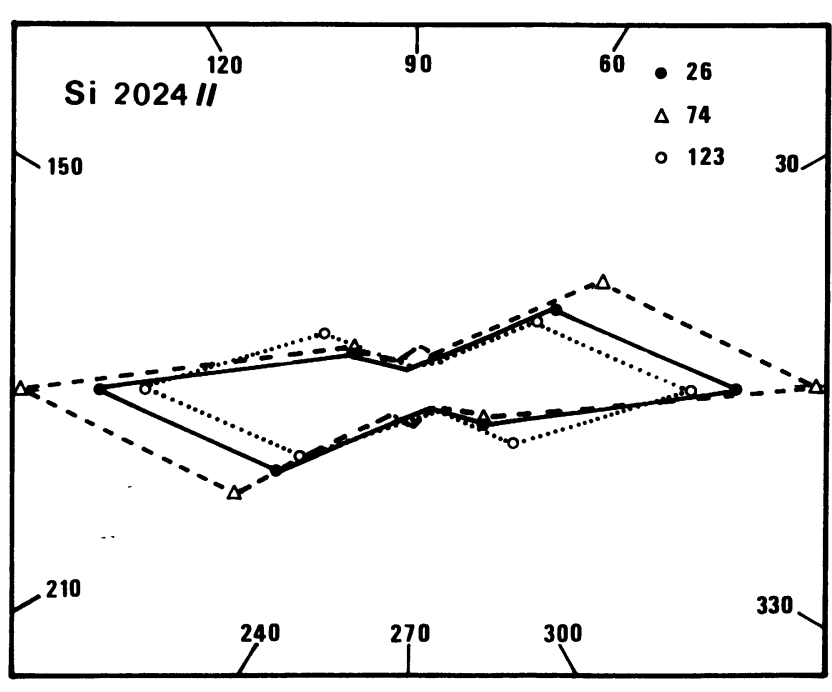

Fig. 5. - Rose des directions dans une section parallèle à l'axe de croissance [4].

[Rose of directions in a parallel cut to the growth axis [4].]

La figure 4 est caractéristique d'une structure isotrope, la rose des directions peut être assimilée à un cercle. Cette représentation correspond à une plaque prélevée dans un bloc d'angle d'un lingot de $55 \mathrm{~kg}$. Il est intéressant de remarquer que les parois du creuset n'ont pas favorisé une croissance préférentielle selon une direction particulière.

La solidification orientée impose une anisotropie très marquée selon l'axe de croissance et de telles 
méthodes d'analyse ne sont évidemment pas nécessaires pour mettre cette anisotropie en évidence. Cependant plusieurs analyses réalisées sur de telles plaques découpées parallèlement à l'axe de croissance ont montré que le gradient thermique vertical lié à l'évacuation de la chaleur latente de solidification n'était pas toujours parallèle à l'axe de croissance (Fig. 5).

3.3 ConClusion. - L'ensemble des analyses granulométriques a permis de quantifier les structures analysées et de définir des conditions de croissance qui conduisent à une structure colonnaire peu perturbée. De plus, cette étude a montré que la stabilisation de la température du bain de silicium était suffisante pour éviter une germination secondaire à partir des parois du creuset. Elle a également confirmé la nécessité d'un dégagement progressif de la surface émissive pour limiter le nombre de germes en début de solidification.

\section{Qualité cristalline.}

La qualité cristalline dépend des conditions de solidification et est un facteur important directement lié aux performances photovoltaïques.

4.1 Attaque chimique. - Le principe de la révélation chimique est basé sur la variation de la vitesse d'attaque du matériau, au voisinage des imperfections cristallines de la surface traitée. Il s'agit de dislocations isolées ou d'arrangement de dislocations. Plusieurs réactifs chimiques sont disponibles, la majorité d'entre eux ont été prévus pour réaliser une attaque préférentielle selon l'orientation cristalline du monocristal [5-7]. Plus récemment, Sopori [8] a mis au point un réactif chimique qui convient pour le silicium polycristallin puisque les résultats sont pratiquement indépendants de l'orientation cristalline. Les dislocations sont révélées sous forme circulaire ou elliptique.

Le but de la caractérisation chimique est de suivre l'évolution de la dualité cristalline au cours de la croissance et de comparer la qualité des lingots afin de corréler les performances photovoltaïques et les conditions d'élaboration.

Les premiers lingots élaborés présentaient un taux de dislocations élevé souvent supérieur ou égal à $10^{5} \mathrm{~cm}^{-2}$. Le taux de dislocations diminuait de bas en haut. La mise au point des paramètres de croissance avec notamment la diminution de la vitesse de solidification dans la partie inférieure du lingot a entraîné une nette amélioration. Une grande partie du lingot a maintenant un taux de dislocations inférieur à $10^{4} \mathrm{~cm}^{-2}$, les zones les plus perturbées correspondent aux zones de fin de solidification, des amas de dislocations peuvent être observées dans le dernier centimètre solidifié (Fig. 6, Tableau III).

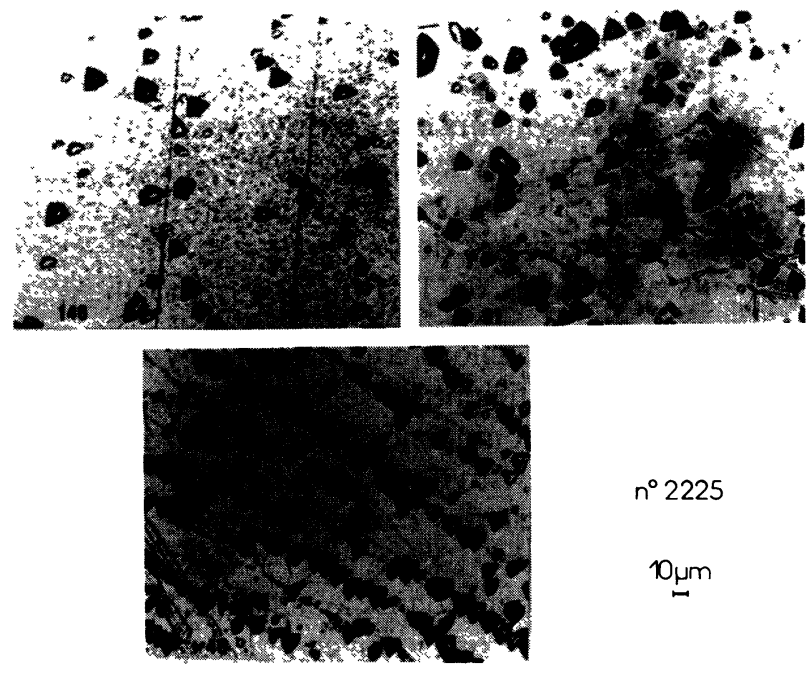

Fig. 6. - Révélation chimique des défauts : évolution le long d'un bloc issu d'un lingot de $55 \mathrm{~kg}$.

[Density of dislocations from the bottom (wafer $\left.n^{\circ} 146\right)$ to the top (wafer $n^{\circ} 40$ ) (ingot 2225).]

Tableau III. - Evolution de la densité de dislocations à l'intérieur d'un lingot de $55 \mathrm{~kg}$.

[Density of dislocations, ingot $2225(55 \mathrm{~kg})$.]

\begin{tabular}{|c|c|c|c|c|c|c|}
\hline & & Bas: & & & Haut & \\
\hline \multirow{2}{*}{$\begin{array}{l}\text { BLOC } \\
\text { CENTRAL }\end{array}$} & $N^{\bullet}$ Pleque & 90 & 80 & 70 & 39 & 10 \\
\hline & $\begin{array}{l}\text { Densité } \\
\text { Dislocations } \mathrm{cm}^{-2}\end{array}$ & $10^{3}$ & $10^{3}$ & $4.10^{3}$ & $8.10^{3}$ & $10^{4}$ \\
\hline BLOC & $N^{\bullet}$ Plaque & 74 & 18 & 4 & & \\
\hline LATERAL & $\begin{array}{l}\text { Densité } \\
\text { Dislocations } \mathrm{cm}^{-2}\end{array}$ & $3.10^{3}$ & $10^{4}$ & $10^{4}$ & & \\
\hline
\end{tabular}

La révélation chimique ne donne qu'une partie de l'information puisqu'elle ne renseigne pas sur l'activité électrique des défauts cristallographiques : dislocations, joints de grains, parois de macle, information qui peut être obtenue par analyse combinée EBIC/TEM [9].

\section{Etat de pureté.}

Les propriétés des matériaux étant très liées à leur pureté, les analyses sont indispensables pour caractériser la méthode d'élaboration ou pour déceler d'éventuelles sources de pollution. La corrélation des résultats des analyses avec les propriétés du matériau conduit ensuite à établir l'influence de la nature des impuretés et à définir des spécifications en vue d'utilisation de silicium de qualité moindre. 


\subsection{ELÉMENTS DE BASE.}

5.1.1 Le silicium utilisé est de qualité électronique ou déclassée, les valeurs des résistivités sont respectivement $450 \Omega \mathrm{cm}$ (type $\mathrm{n}$ ) - $5000 \Omega \mathrm{cm}$ (type p), $50 \Omega \mathrm{cm}$ (type $\mathrm{n}$ ) $-500 \Omega \mathrm{cm}$ (type p) soit des teneurs en éléments donneurs de $10^{13}$ à $10^{14}$ at. $\mathrm{cm}^{-3}$ et en éléments accepteurs de $10^{12}$ à $2 \times 10^{13}$ at. $\mathrm{cm}^{-3}$.

5.1.2 Le bore a été choisi comme élément dopant en raison de son coefficient de ségrégation qui favorise une distribution homogène à l'intérieur du lingot. L'intervalle de résistivité a été fixé entre 0,5 et $2 \Omega \mathrm{cm}$ (type p) soit des concentrations en élément dopant voisines de $10^{16}$ at. $\mathrm{cm}^{-3}$. Les impuretés présentes initialement dans le silicium sont donc négligeables par rapport au niveau de dopage choisi.

5.1.3 L'agent d'encapsulation est utilisé comme barrière de diffusion des impuretés du creuset dans le bain de silicium. Son efficacité a été démontrée aux Laboratoires de Marcoussis en comparant les teneurs en impuretés des lingots élaborés avec et sans agent d'encapsulation. Le tableau IV donne quelques résultats issus d'échantillons prélevés dans des lingots de $15 \mathrm{~kg}[10]$.

Tableau IV. - Efficacité de l'encapsulation.

[Efficiency of the encapsulation.]

\begin{tabular}{|l|c|c|c|c|}
\hline \multirow{2}{*}{$\begin{array}{l}\text { Eléments } \\
\text { atem }\end{array}$} & \multicolumn{2}{|c|}{$\begin{array}{c}\text { Lingot } \\
\text { encapsulation }\end{array}$} & \multicolumn{2}{c|}{$\begin{array}{c}\text { Lingot avec } \\
\text { encapsulation }\end{array}$} \\
\cline { 2 - 5 } & haut & milieu & haut & milleu \\
\hline $\mathrm{Cr} \times 10^{12}$ & 12 & $<0,8$ & 4 & 2 \\
\hline $\mathrm{F} \times 10^{14}$ & 26 & $<0,25$ & $<1,6$ & $<1$ \\
\hline $\mathrm{Zr} \times 10^{13}$ & 12 & 20 & $<6$ & $<4,6$ \\
\hline $\mathrm{Ag} \times 10^{12}$ & 6,5 & 1 & 0,95 & 1,8 \\
\hline
\end{tabular}

5.1.4 Le creuset en graphite peut être en cas de détérioration du film d'encapsulation une source de carbone et d'éléments métalliques, le graphite utilisé est garanti à moins de $0,3 \%$ en poids de cendres. Les lingots sont élaborés sous pression partielle d'argon.

5.2 Méthodes D’ANAlyses. - En raison de la faible teneur en impuretés et de leur influence sur les propriétés du matériau, il est nécessaire d'utiliser des méthodes d'analyse à faible limite de détection. L'analyse par activation de particules chargées est utilisée du fait de sa grande sensibilité, de la possibilité d'analyser des échantillons massifs, jusqu'à un centimètre cube, et de détecter pratiquement tous les types d'impuretés. Les analyses ont été effectuées à Orléans dans le laboratoire de Mr Debrun, service du cyclotron. Les éléments dosés ont été choisis en fonction de leur influence éventuelle sur les propriétés du silicium (Tableau V).

Des dosages plus systématiques du carbone et de l'oxygène sont réalisés par spectroscopie infrarouge. Seuls sont mesurés, les atomes d'oxygène interstitiels et les atomes de carbone substitutionnels. La présence de précipités étant rarement décelée et la méthode ayant été étalonnée à l'aide d'échantillons analysés par activation de particules chargées, les 'résultats obtenus sont donc valables. La limite de détection du carbone varie de $10^{16}$ at. $\mathrm{cm}^{-3}$ à température ambiante à $3 \times 10^{15}$ at. $\mathrm{cm}^{-3}$ à $77 \mathrm{~K}$, celle de l'oxygène est voisine de $10^{15}$ at. $\mathrm{cm}^{-3}$. Les analyses sont effectuées à l'Université de Caen dans le laboratoire de Mr Lavalley, les appareils sont des spectromètres infrarouges à transformée de Fourier de type NICOLET MX1 ou 10MX.

Tableau V. - Eléments dosés et méthodes utilisées.

[Elements analysed and methods chosen.]

\begin{tabular}{|c|c|}
\hline Eléments dosés & Méthodes ut1lisées \\
\hline $\begin{array}{l}\text { B, Cu, N1, Cr, } \\
\text { Fe, Zr, Co, Ti } \\
\text { Ge, Mn, Ca }\end{array}$ & $\begin{array}{l}\text { Protone de } 20 \mathrm{MeV} \\
\text { Mesures spectrométrie gamme haute résolution } \\
\text { evec un détecteur Ge(L1) } \\
\text { Seneibilité de } 10^{12} \text { a } 10^{15} \text { at. } \mathrm{cm}^{-3} \text { selon lee éléments }\end{array}$ \\
\hline Oxygène & $\begin{array}{l}\text { Tritona de } 3,5 \mathrm{MeV} \\
\text { euivant la réaction } 16 \mathrm{o}(t, \mathrm{n})^{18} \mathrm{~F} \\
\text { seneibilité } 10^{14} \text { at. } \mathrm{cm}^{-3}\end{array}$ \\
\hline Carbone & $\begin{array}{l}\text { Deutons de } 3 \mathrm{MeV} \\
12 \mathrm{c}(\mathrm{d}, \mathrm{n})^{13} \mathrm{~N} \\
\text { sensibilité de } 10^{15} \text { at. } \mathrm{cm}^{-3}\end{array}$ \\
\hline
\end{tabular}

5.3 Résultats. - Plusieurs séries d'échantillons ont été prélevés à différentes hauteurs et positions à l'intérieur de lingots de 15 à $30 \mathrm{~kg}$ en vue d'étudier la ségrégation et l'état de pureté du matériau.

5.3.1 Le dosage du bore. - L'évolution de la concentration en bore selon la hauteur du lingot est présentée dans la figure 7 . On note une augmentation de la concentration vers le haut du lingot. A un même niveau, les échantillons prélevés à proximité des parois du creuset ont une concentration légèrement plus. faible que ceux prélevés au centre du lingot. La variation de la concentration peut également être estimée à partir de la mesure de la résistivité. La résistivité de la majorité des lingots est comprise entre 0,8 et $1,5 \Omega \mathrm{cm}$. Cette variation est indiquée pour le même lingot sur la figure 8 .

Les profils de résistivité sont établis en mesurant la résistivité quatre pointes sur trois zones de plaques 


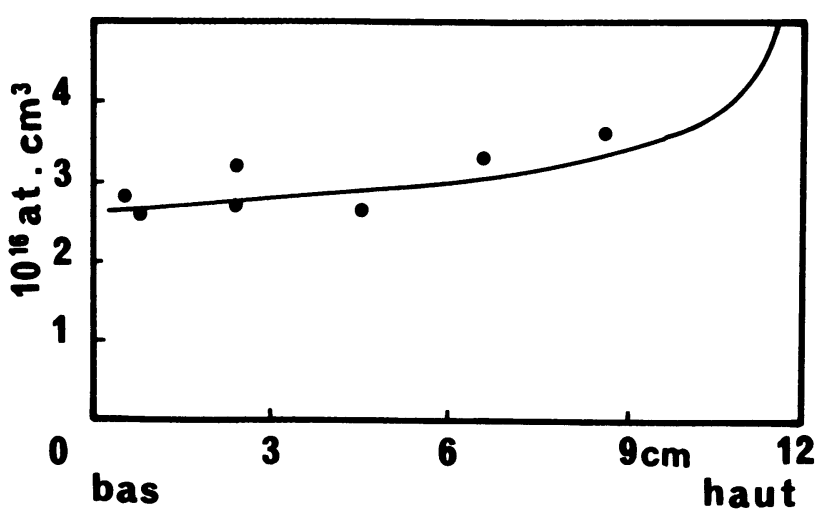

Fig. 7. - Concentration en bore dans un échantillon analysé par activation des particules chargées. Courbe calculée à partir de l'équation (lingot 1149): $C=$ $C_{0} k(1-g)^{k-1}$ avec $k=0,8, C=2,6 \times 10^{16}$ at. $\mathrm{cm}^{-3}$.

[Bore concentration in a sample analysed by activation of charged particles. The curve is calculated from the equation (ingot 1149) $C=C_{0} k(1-g)^{k-1}, k=0.8, C=$ $2.6 \times 10^{16}$ at. $\mathrm{cm}^{-3}$.]

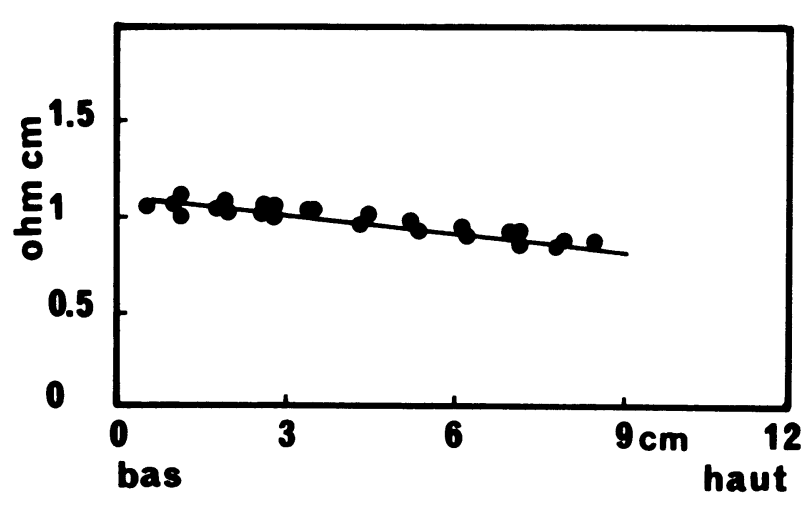

Fig. 8. - Variation de la résistivité (lingot 1149).

[Plot of the resistivity (ingot 1149).]

prélevées tous les centimètres selon la hauteur du lingot. La dispersion des valeurs mesurées perpendiculairement à l'axe de croissance est du même ordre de grandeur que la variation enregistrée selon la hauteur (Fig. 9).

5.3.2 Le dosage d'autres éléments. - Il faut tout d'abord noter le très bon état de pureté des échantillons. La limite de détection de nombreuses impuretés n'est pas toujours atteinte. Le fer, le manganèse et le nickel sont les impuretés métalliques dominantes dans le lingot 1149. L'évolution des teneurs montre pour ce lingot un enrichissement dans la partie supérieure (Tableau VI). La comparaison entre les concentrations mesurées à la périphérie et au centre du bloc ne met pas en évidence une distinction dans la répartition.

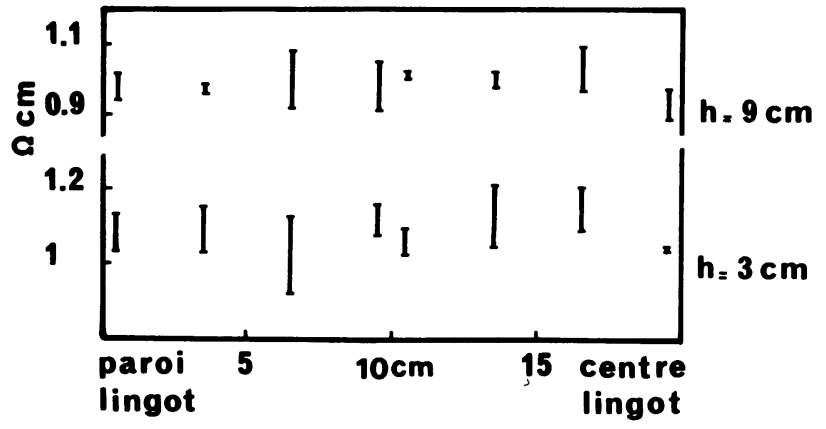

Fig. 9. - Demi-coupe verticale d'un lingot $(50 \mathrm{~kg})$ : dispersion horizontale de la résistivité. Chaque trait correspond à la dispersion de la résistivité mesurée dans une zone d'un centimètre carré située sur une ligne horizontale du lingot à $3 \mathrm{~cm}$ et à $9 \mathrm{~cm}$ du bas du lingot.

[Vertical half part of a $50 \mathrm{~kg}$ ingot : horizontal deviation of the resistivity. Each bar corresponds to the deviation of the resistivity for one $\mathrm{cm}^{2}$ area. The measurements are carried out on a horizontal line at $3 \mathrm{~cm}$ and $9 \mathrm{~cm}$ from the bottom of the ingot.]

Tableau VI. - Résultats d'analyse par activation lingot $1149(15 \mathrm{~kg})$.

[Analyses by activation, ingot $1149(15 \mathrm{~kg})$.]

\begin{tabular}{|c|c|c|c|c|c|c|c|c|c|c|c|c|}
\hline \multicolumn{2}{|c|}{$\begin{array}{l}\text { Impureté } \\
\text { Echent11lon }\end{array}$} & $\begin{array}{c}B \\
\times 10^{16}\end{array}$ & Ca & TI & $C_{r}$ & \multicolumn{2}{|c|}{$x_{10} 10^{12}$ et.c } & $\begin{array}{r}\text { Co } \\
. \mathrm{cm}^{-3}\end{array}$ & N1 & Cu & $2 n$ & Ge \\
\hline HAUT & $2 c$ & 3,6 & 45000 & 30 & 400 & $<30$ & 12000 & $<60$ & 48 & 800 & $<60$ & $<5$ \\
\hline & $27 \mathrm{C}$ & 3,3 & $<2$ & $<2$ & $<3$ & 17 & $<9$ & 40 & 28 & 120 & $<3$ & 6,7 \\
\hline & 278 & 4,5 & 17000 & $<1000$ & 1900 & 8 & 23000 & $8 \infty$ & 240 & 8800 & $<600$ & $\infty$ \\
\hline & $53 \mathrm{C}$ & 2,6 & $<2$ & $<6$ & $<6$ & 26 & 80 & $<30$ & $<40$ & $<40$ & $<4$ & 5,3 \\
\hline & $53 E$ & 2,4 & $<4$ & $<10$ & $<15$ & 50 & $<40$ & $<50$ & 11 & $<60$ & $<7$ & 6,7 \\
\hline & $79 \mathrm{C}$ & 3,2 & $<2$ & $<4$ & $<4$ & 23 & $<0$ & $<20$ & 3,8 & $<30$ & $<3$ & 6,4 \\
\hline & $79 E$ & 2,7 & $<2$ & $<5$ & $<6$ & 30 & 60 & $<30$ & 18 & $<30$ & $<4$ & 3,5 \\
\hline & $97 \mathrm{C}$ & 2,6 & $<4$ & $<9$ & $<10$ & & 500 & $<40$ & 6,5 & $<50$ & $<6$ & $<$ \\
\hline & $97 E$ & 1,9 & $<10$ & $<5$ & $<6$ & 25 & 250 & $<30$ & 20 & $<60$ & $<10$ & $<$ \\
\hline & $98 \mathrm{C}$ & 2,8 & 100 & $<7$ & 13 & $<9$ & 1500 & $<20$ & $<1$ & $<30$ & $<10$ & 5 \\
\hline BAS & $98 E$ & 2 & $<8$ & $<6$ & $<8$ & 40 & 450 & $<30$ & 20 & $<50$ & $<3$ & 5 \\
\hline
\end{tabular}

Inférieur : : IImite de détection non atteinte,

* : échantillon poreux,

E : échantillon prélevé à proximité de la surface latérale du lingot,

c : échantillon prélevé à proximité du coeur du lingot.

5.3.3 Carbone et oxygène. - Des échantillons prélevés à l'intérieur de lingots de $15 \mathrm{~kg}$ élaborés au début de l'étude ont été analysés par activation. Les résultats sont présentés dans le tableau VII. La concentration d'oxygène a tendance à décroître du bas vers le haut du lingot. Les concentrations sont élevées et voisines de $10^{18} \mathrm{at} . \mathrm{cm}^{-3}$. La dispersion des résultats concernant le carbone est importante, les concentrations les plus élevées sont mesurées au centre de la partie inférieure du lingot. Les résultats qualitatifs et comparatifs obtenus par spectroscopie infrarouge confirment ces analyses. 
Tableau VII. - Evolution de la concentration en carbone et oxygène, lingots 1068 et 1069.

[Carbon and oxygen concentration, ingot 1068 and 1069.]

\begin{tabular}{|c|c|c|c|c|}
\hline LINGOT & \multicolumn{2}{|c|}{$\begin{array}{l}\text { Position } \\
\text { échantillon }\end{array}$} & $\begin{array}{c}\text { Teneur en oxygène } \\
10^{17} \mathrm{at}^{\mathrm{c} . \mathrm{cm}^{-3}}\end{array}$ & $\begin{array}{c}\text { Teneur en carbone } \\
10^{17}{\mathrm{at} . \mathrm{cm}^{-3}}^{-3}\end{array}$ \\
\hline \multirow{4}{*}{1069} & \multirow{2}{*}{ Bea } & extérieur & 8,6 & 4,7 \\
\hline & & intérieur & 10,3 & 14,2 \\
\hline & \multirow{2}{*}{ Haut } & extérieur & 8,3 & 4,3 \\
\hline & & Intérieur & 5,8 & 5,9 \\
\hline \multirow{3}{*}{1068} & \multirow{2}{*}{ Bas } & extérieur & 10,6 & 5 \\
\hline & & Intérieur & 12,5 & 9,4 \\
\hline & Haut & Intérieur & 9 & 5,5 \\
\hline \multicolumn{3}{|c|}{ Incertitude } & $10 \%$ & $20 \%$ \\
\hline
\end{tabular}

5.4 COEFFICIENTS DE SÉGRÉGATION. - Les résultats obtenus sont comparés avec la concentration des impuretés calculées à partir de la loi de ségrégation :

$$
C=C_{0} k(1-g)^{k-1}
$$

avec $g$ la fraction solidifiée.

En ce qui concerne le bore, les courbes tracées pour des valeurs de $k$ égales à 0,8 et 0,9 montrent la conformité des résultats pour le lingot 1149 (Fig. 7).

La présence de phosphore dans un lingot se traduit par une augmentation importante de la résistivité dans la partie supérieure du lingot. Le coefficient de ségrégation estimé à partir des mesures de résistivité et des teneurs analysées est voisin de 0,6 contre 0,35 dans la littérature pour le silicium monocristallin [11].

L'oxygène a un coefficient de ségrégation supérieur à 1 , une concentration supérieure dans la partie basse du lingot est systématiquement observée. Quelques analyses conduisent à des valeurs du coefficient de ségrégation de 1,1 à 1,35 , toutefois tous les points ne sont pas pris en compte car il existe une dispersion importante.

Les résultats des concentrations en carbone diffèrent suivant les lingots, il n'y a pas systématiquement augmentation de la teneur en carbone sur le haut du lingot, résultat attendu du fait de la valeur du coefficient de ségrégation voisine de $10^{-4}$. Le creuset agit comme source de carbone mais l'effet est limité du fait de l'encapsulation.

5.5 INTERPRÉTATION. - L'encapsulation joue un rôle important vis-à-vis de la pureté du lingot élaboré. Le rôle de barrière de diffusion des impuretés du creuset vers le silicium a été vérifié. Au cours de l'étude, il a été montré la nécessité d'utiliser un agent d'encapsulation de très bonne qualité chimique avec notamment de faibles teneurs en éléments métalliques ou en éléments dopants.

Les quelques analyses effectuées concernant les autres éléments ne permettent pas d'accéder avec précision aux coefficients de ségrégation. Toutefois, les résultats obtenus sur les lingots élaborés à Caen et aux Laboratoires de Marcoussis conduisent à des coefficients de ségrégation dix à cent fois supérieurs à ceux de la littérature (Tableau VIII). La répartition des teneurs en carbone n'est pas toujours conforme à celle attendue, il existe en effet pour certains lingots un enrichissement à proximité des parois ou du fond du creuset, l'encapsulation n'est donc pas toujours parfaite.

Tableau VIII. - Comparaison des coefficients de ségrégation effectifs du Polix avec les valeurs de Bathey et Cretella [12].

[Comparison of the effectif segregation coefficients of the Polix with the values of Bathey and Cretella [12].]

\begin{tabular}{|c|c|c|}
\hline ELEMENTS & $\begin{array}{c}\mathrm{K}_{\text {eff }} \text { Polix } \\
\times 10^{-5}\end{array}$ & $\begin{array}{c}\mathrm{K}_{0} \neq \text { equilib. } \\
\times 10^{-5}\end{array}$ \\
\hline $\mathrm{Cu}$ & 500 & 40 \\
\hline $\mathrm{As}$ & 9800 & 30000 \\
\hline $\mathrm{Na}$ & 4700 & 200 \\
\hline $\mathrm{Zn}$ & 9,4 & 1 \\
\hline $\mathrm{Fe}$ & 14 & 0,8 \\
\hline $\mathrm{Co}$ & 14 & 1 \\
\hline $\mathrm{Ni}$ & 480 & 10 \\
\hline $\mathrm{Cr}$ & 100 & 1 \\
\hline
\end{tabular}

Cependant, pour la plupart des lingots, la résistivité obtenue est en accord avec celle prévue, les limites de détection de nombreuses impuretés ne sont pas atteintes, les propriétés photovoltaïques sont bonnes, l'état de pureté est donc satisfaisant. Ceci est principalement dû à la qualité du silicium de départ et au fait que la méthode d'élaboration n'affecte pas la qualité chimique du matériau. La 
solidification dirigée permet la ségrégation d'un grand nombre d'impuretés, ceci est un atout pour envisager dans un proche avenir l'utilisation de silicium de qualité moindre.

\section{Influence de la qualité cristalline et de l'état de pureté sur les propriétés photovoltaïques.}

6.1 InTRODUCTION. - La finalité de l'élaboration des lingots est la production de matériau destiné à la réalisation de cellules photovoltaïques. Les performances photovoltaïques constituent donc la caractérisation la plus importante du matériau. Sur chaque lingot élaboré, une série de plaques est prélevée et

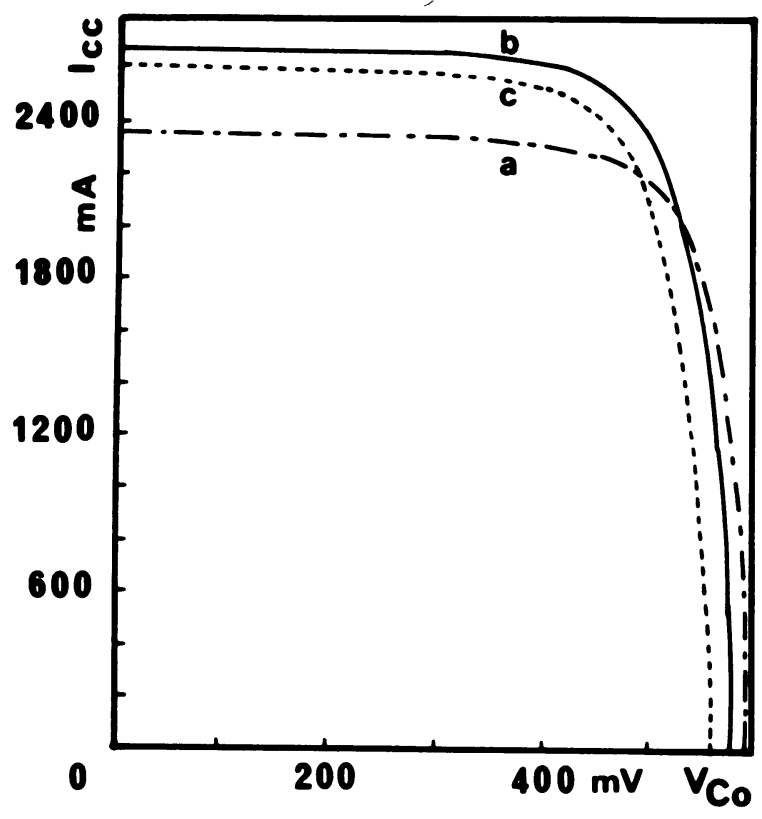

Fig. 10. - Courbes courant-tension de cellules solaires en silicium monocristallin (a), Polix (b) et Silso (c).

[Intensity potential plots of solar cells, a) monocrystalline, b) Polyx, c) Silso.]

Tableau IX. - Caractéristiques de trois cellules représentatives.

[Photovoltaic characteristics of three typical solar cells.]

\begin{tabular}{|l|c|c|c|c|c|c|c|}
\hline Matér1au & $\begin{array}{c}\mathrm{J}_{\text {sc }}-2 \\
\mathrm{~mA}^{-2}\end{array}$ & $\begin{array}{c}\mathrm{V}_{\text {oc }} \\
\mathrm{mV}\end{array}$ & $\begin{array}{c}\mathrm{I}_{\mathrm{M}} \\
\mathrm{mA}\end{array}$ & $\begin{array}{c}\mathrm{V}_{\mathrm{M}} \\
\mathrm{mV}\end{array}$ & $\begin{array}{c}\mathrm{P}_{\max } \\
\mathrm{mh}\end{array}$ & $\mathrm{FF}$ & $\begin{array}{c}\text { Rend. } \\
\%\end{array}$ \\
\hline $\begin{array}{l}\text { POLIX } \\
100 \mathrm{~cm}^{2}\end{array}$ & 27 & 577 & 2428 & 472 & 1146 & 74 & 11,5 \\
\hline $\begin{array}{l}\text { MONO } \\
78,5 \mathrm{~cm}^{2}\end{array}$ & 31 & 590 & 2178 & 499 & 1082 & 76 & 13,8 \\
\hline $\begin{array}{l}\text { SILSO } \\
100 \mathrm{~cm}^{2}\end{array}$ & 26,5 & 557 & 2430 & 446 & 1084 & 73 & 10,8 \\
\hline
\end{tabular}

caractérisée électriquement. Les cellules sont réalisées selon le procédé standard Photowatt. Les paramètres mesurés sont (Fig. 10) (Tableau IX) :

- la tension en circuit ouvert $V_{\mathrm{co}}$,

- le courant de court-circuit $J_{\mathrm{sc}}$,

- le facteur de forme de la caractéristique $F F$,

- les valeurs maximums de la tension et du courant,

- le rendement photovoltaique $\eta$.

Les propriétés photovoltaïques sont directement liées à l'état de pureté du matériau et à la qualité cristalline, paramètres dépendant des conditions de solidification. L'amélioration des conditions de croissance entraîne donc l'amélioration des performances du matériau.

Les corrélations établies entre le rendement photovoltaïque et les caractérisations du matériau ont permis la mise en évidence des facteurs limitatifs. Les conditions d'élaboration ont alors été adaptées pour tenter de résoudre les problèmes posés.

6.2 EVOlution DES PERFORMANCES. - Le premier facteur analysé est le rendement photovoltaïque. Au début de l'étude, le rendement maximum était voisin de $9 \%$ et la dispersion des résultats en fonction de la position de la cellule à l'intérieur du lingot était importante. Rapidement une nouvelle composition de l'agent encapsulant a été testée, il en a résulté une amélioration sensible des performances : environ 1 point de rendement (Fig. 11).

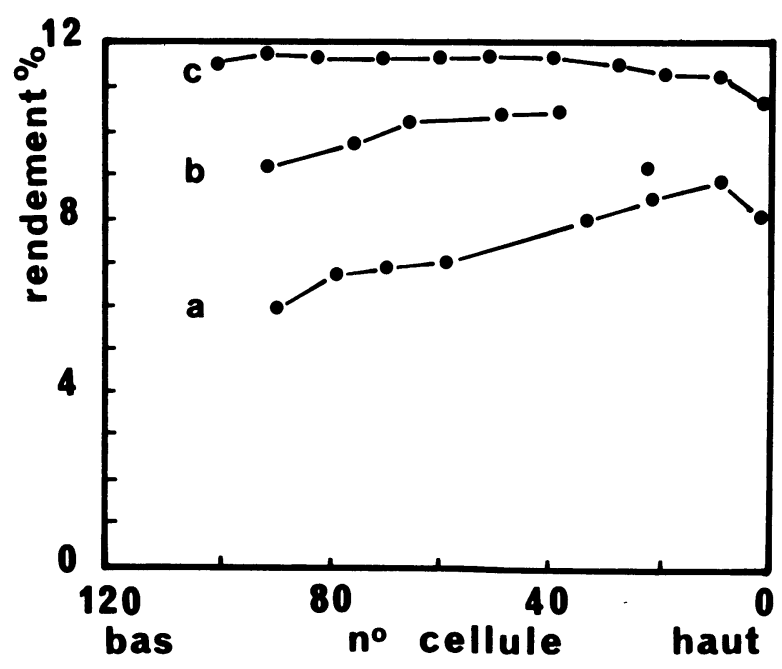

Fig. 11. - Evolution des performances le long d'un lingot au cours de l'étude : a) lingot $13 \mathrm{~kg}$ au début de l'étude ; b) lingot $13 \mathrm{~kg}$ après modification de la composition de l'encapsulant liquide et adaptation des paramètres de solidification; c) lingot $55 \mathrm{~kg}$ après optimisation des conditions d'élaboration.

[Variation of the photovoltaic yield along the growth axis : a) $13 \mathrm{~kg}$ ingot, beginning ; b) $13 \mathrm{~kg}$ ingot after modification of the chemical composition of the liquid encapsulant ; c) $55 \mathrm{~kg}$ ingot after optimization.] 
Cette amélioration a été obtenue par une meilleure encapsulation d'où une efficacité supérieure dans son rôle de barrière anti-diffusion des impuretés $\mathrm{du}$ creuset vers le silicium, et par une meilleure pureté des produits utilisés pour élaborer le sel d'encapsulation.

L'analyse des propriétés photovoltaïques en fonction de la position des plaques à l'intérieur d'un lingot avait mis en évidence une augmentation des performances maximales obtenues sur des plaques issues des blocs périphériques. Ces résultats ont été corrélés avec l'évolution de la qualité cristalline et de la morphologie du matériau; les meilleures performances coïncident avec la meilleure qualité cristalline. Simultanément, il n'était pas noté d'évolution sensible de la distribution des impuretés. Les conditions de solidification ont donc été adaptées : la descente progressive de l'isolation thermique face à la surface émissive a entraîné une diminution de la vitesse de solidification, d'où une amélioration de la qualité cristalline dans la partie inférieure du lingot. Ceci s'est traduit par une diminution dans la dispersion verticale des résultats photovoltaïques.

Les performances photovoltaïques obtenues avec les lingots élaborés selon le procédé standard sont satisfaisantes. Les principaux résultats 1985/1986 sont présentés figure 12 .

$\mathrm{La}$ dispersion résiduelle est due essentiellement :

- dans la partie inférieure du lingot, à une concentration en oxygène plus élevée, liée à la
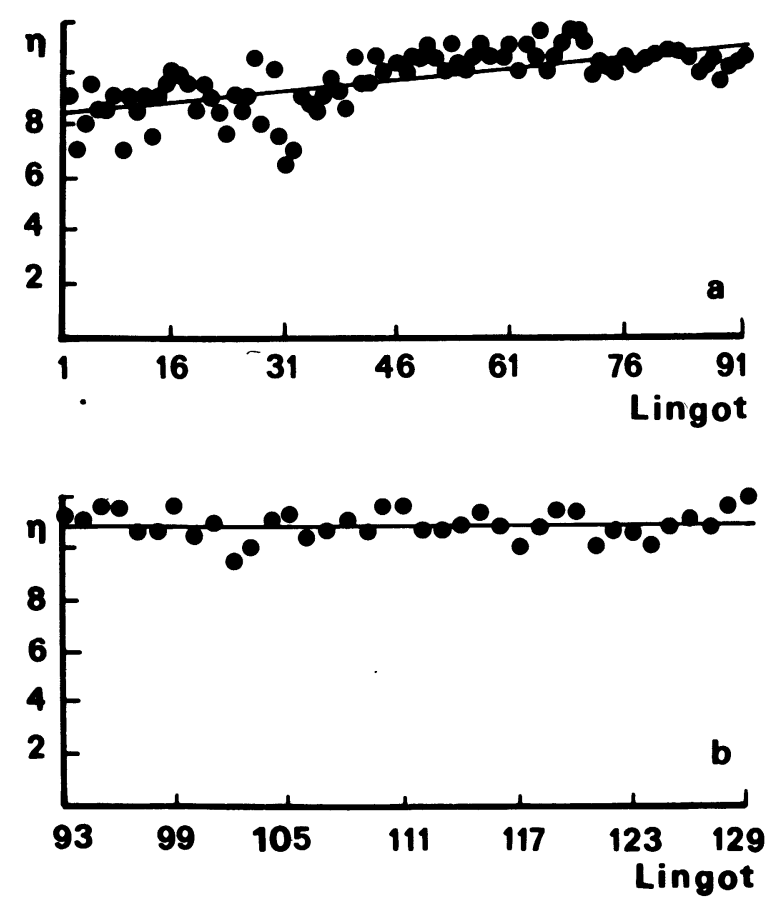

Fig. 12. - Rendements de conversion moyens : a) 1985 ; b) 1986 .

[Mean photovoltaic yield : a) 1985 ; b) 1986.] présence de l'encapsulant, de nouvelles compositions d'encapsulant sont à l'étude ;

- dans la partie supérieure, sur le dernier centimètre solidifié ou dans les angles sur deux ou trois centimètres, à la structure cristalline présentant un nombre de défauts : dislocations, dendrites et porosités liées à des difficultés de fin de solidification.

6.3 RÉsultats Quantitatifs. - Il est difficile de quantifier les corrélations entre certaines propriétés du matériau, taux de dislocations, état de pureté et les performances photovoltaïques; les différents paramètres étant souvent étroitement liés. Toutefois, il est possible de définir des spécifications suffisantes sur la qualité du matériau pour obtenir des performances satisfaisantes. La validité de ces lois est $a$ priori limitée au Polix élaboré avec du silicium de qualité électronique ou électronique dégradée.

\subsubsection{Influence de la qualité cristalline et de la teneur} en carbone et oxygène. - Si le taux de dislocations est inférieur à $10^{4} \mathrm{~cm}^{-2}$ et si les teneurs en oxygène et en carbone sont respectivement inférieures à $6 \times 10^{17}$ et $5 \times 10^{17}$ at $\mathrm{cm}^{-3}$, le rendement photovoltaïque est supérieur à $11 \%$.

Si le taux de dislocations est supérieur à $10^{5} \mathrm{~cm}^{-2}$, le rendement photovoltaïque est systématiquement inférieur à $10 \%$.

Pour les autres cas, les paramètres sont liés et d'autres phénomènes interviennent tel le traitement thermique subi par le lingot. A titre d'exemple, le rendement photovoltaïque en fonction des teneurs en oxygène et carbone d'une centaine de plaques prélevées à l'intérieur de lingots différents sont présentés figure 13. Il ne se dégage pas de loi des résultats présentés. Dans cette zone intermédiaire, les teneurs en carbone ou en oxygène ne sont pas déterminantes.

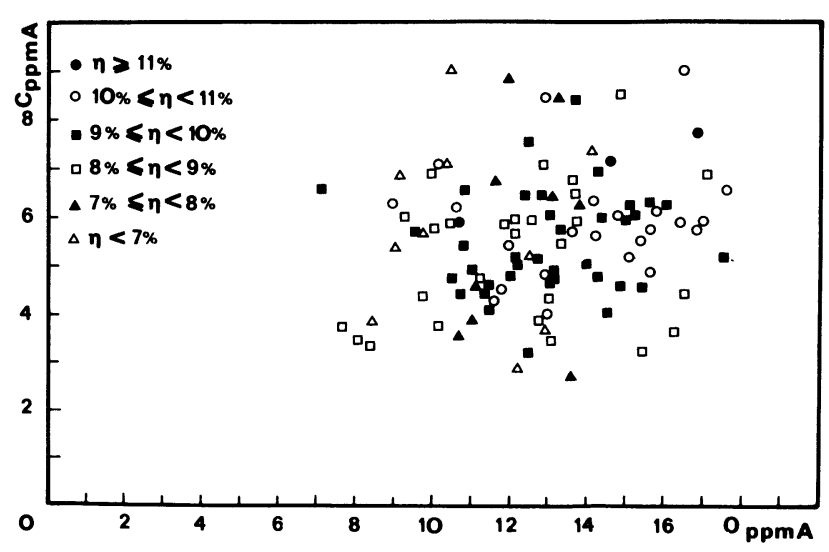

Fig. 13. - Rendement photovoltaïque en fonction de la teneur en carbone et oxygène.

[Photovoltaic efficiency versus carbon and oxygen concentration.] 
6.3.2 Influence du dopage. - Avec la qualité de silicium utilisée, il est possible de fixer le dopage entre 0,1 et $5 \Omega \mathrm{cm}$. Les résultats obtenus au cours de l'étude permettent de définir les tendances quant à l'influence de la résistivité sur les propriétés photovoltaïques. Il ne s'agit que de tendance car il y a interaction avec les paramètres présentés au paragraphe précédent et l'examen des résultats ne permet pas d'établir une loi systématiquement vérifiée.

Des investigations ont été menées à partir de quatre-vingt essais balayant un intervalle de résistivité de 0,1 à $3,85 \Omega \mathrm{cm}$. La méthode utilisée est le «tribaguette» qui permet de classer suivant un paramètre croissant les autres paramètres et de visualiser d'éventuelles corrélations.

Il en ressort que les valeurs élevées du courant de court-circuit correspondent aux valeurs élevées de résistivité. La plus grande valeur de la tension de circuit ouvert $V_{\text {co }}(603 \mathrm{mV})$ correspond à la plus faible valeur de résistivité $(0,1 \Omega \mathrm{cm})$. La variation de la résistivité de 0,8 à $3 \Omega \mathrm{cm}$ affecte donc peu la valeur de $V_{\text {co. }}$. Ainsi du fait de l'accroissement du courant de court-circuit, il en résulte que les résistivités supérieures à $1,5 \Omega \mathrm{cm}$ conduisent à des rendements supérieurs à $11 \%$. Une difficulté apparaît pour des valeurs supérieures à $2 \Omega \mathrm{cm}$, la résistivité devient alors sensible à l'introduction d'éléments de type $n$. En cas de pollution, le type du lingot est directement inversé et le lingot est alors inexploitable.

Tableau X. - Spécifications chimiques conditionnant l'utilisation de silicium de qualité moindre.

[Chemical specifications limiting the use of lowergrade silicon.]

\begin{tabular}{|c|c|c|c|c|}
\hline \multirow[t]{2}{*}{ ELEMENTS } & \multicolumn{2}{|c|}{ 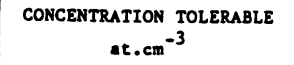 } & \multicolumn{2}{|c|}{$\begin{array}{l}\text { CONCENTRATION TOLERABLE } \\
\text { at. } \mathrm{cm}^{-3}\end{array}$} \\
\hline & tranche & charge & tranche & charge \\
\hline Al & $3.10^{16}$ & $1,5.10^{17}$ & $1.10^{17}$ & $5.10^{17}$ \\
\hline B & $9.10^{15}$ & $7,8.10^{15}$ & $1,5.10^{16}$ & $1,2 \cdot 10^{16}$ \\
\hline Co & $7.10^{14}$ & $8,7.10^{15}$ & $1.10^{15}$ & $1,2 \cdot 10^{16}$ \\
\hline In & $7.10^{14}$ & $1,7.10^{16}$ & $1.10^{15}$ & $2,6 \cdot 10^{16}$ \\
\hline $\mathbf{P}$ & $7.10^{14}$ & $4,2.10^{14}$ & $1.10^{15}$ & $6.10^{14}$ \\
\hline A* & $7.10^{14}$ & $4,5.10^{14}$ & $1.10^{15}$ & $6,5 \cdot 10^{14}$ \\
\hline Sb & $7.10^{14}$ & $1,9.10^{15}$ & $1.10^{15}$ & $2,7.10^{15}$ \\
\hline $\mathrm{Cu}$ & $7.10^{15}$ & $1,4.10^{17}$ & $1,9.10^{16}$ & $3,8 \cdot 10^{17}$ \\
\hline Cr & $2,5.10^{14}$ & $8,2.10^{16}$ & $1.10^{15}$ & $3.10^{17}$ \\
\hline $\mathrm{Fe}$ & $6.10^{14}$ & $4,2.10^{17}$ & $2,6.10^{15}$ & $1,85 \cdot 10^{18}$ \\
\hline N1 & $1,2.10^{17}$ & $2,5.10^{18}$ & $3,3.10^{17}$ & $6,9 \cdot 10^{18}$ \\
\hline Mo & $1.10^{13}$ & $10^{16}$ & $2,8 \cdot 10^{13}$ & $3.10^{16}$ \\
\hline $2 x$ & $4.10^{14}$ & $1,5.10^{17}$ & $1,5 \cdot 10^{15}$ & $6.10^{17}$ \\
\hline c & $5.10^{17}$ & $3,6.10^{17}$ & $6.10^{17}$ & $4,4 \cdot 10^{18}$ \\
\hline 0 & $6.10^{11}$ & $1,3.10^{19}$ & $8,4 \cdot 10^{17}$ & $1,9.10^{19}$ \\
\hline
\end{tabular}

(1)

(2)

(1) Spécifications chimiques pour avoir $\mathrm{J}_{\mathrm{sc}}>27 \mathrm{~mA} \cdot \mathrm{cm}^{-2}(n>11,5 \%)$ sur 90\% du lingot (procédé actue1).

(2) Spécifications chimiques pour avoir $\mathrm{J}_{\mathrm{sc}}>25 \mathrm{~mA} \cdot \mathrm{cm}^{-2}(n>9,5 \%)$ sur 90\% du lingot (procédé actuel).
Avec une résistivité centrée entre 1 et $1,5 \Omega \mathrm{cm}$, une pollution entraîne une dérive de la résistivité sans inversion de type et il est alors possible d'en déceler la cause en évitant la perte de lingot. Il s'agit donc de l'intervalle de valeurs choisi au stade actuel du développement.

Il faut noter que de bons résultats ont été obtenus avec une résistivité de $1 \Omega \mathrm{cm}$ ce qui confirme l'interaction avec d'autres paramètres.

6.3.3 Spécifications chimiques. - Des spécifications chimiques ont été établies aux Laboratoires de Marcoussis concernant l'état de pureté en vue de l'utilisation de silicium de qualité moindre [10]. Ces résultats sont présentés dans le tableau $\mathrm{X}$. La dégradation des performances en fonction de la qualité chimique ainsi que la comparaison avec le comportement du silicium monocristallin sont présentées sur les figures $14 a$ et $14 b$.
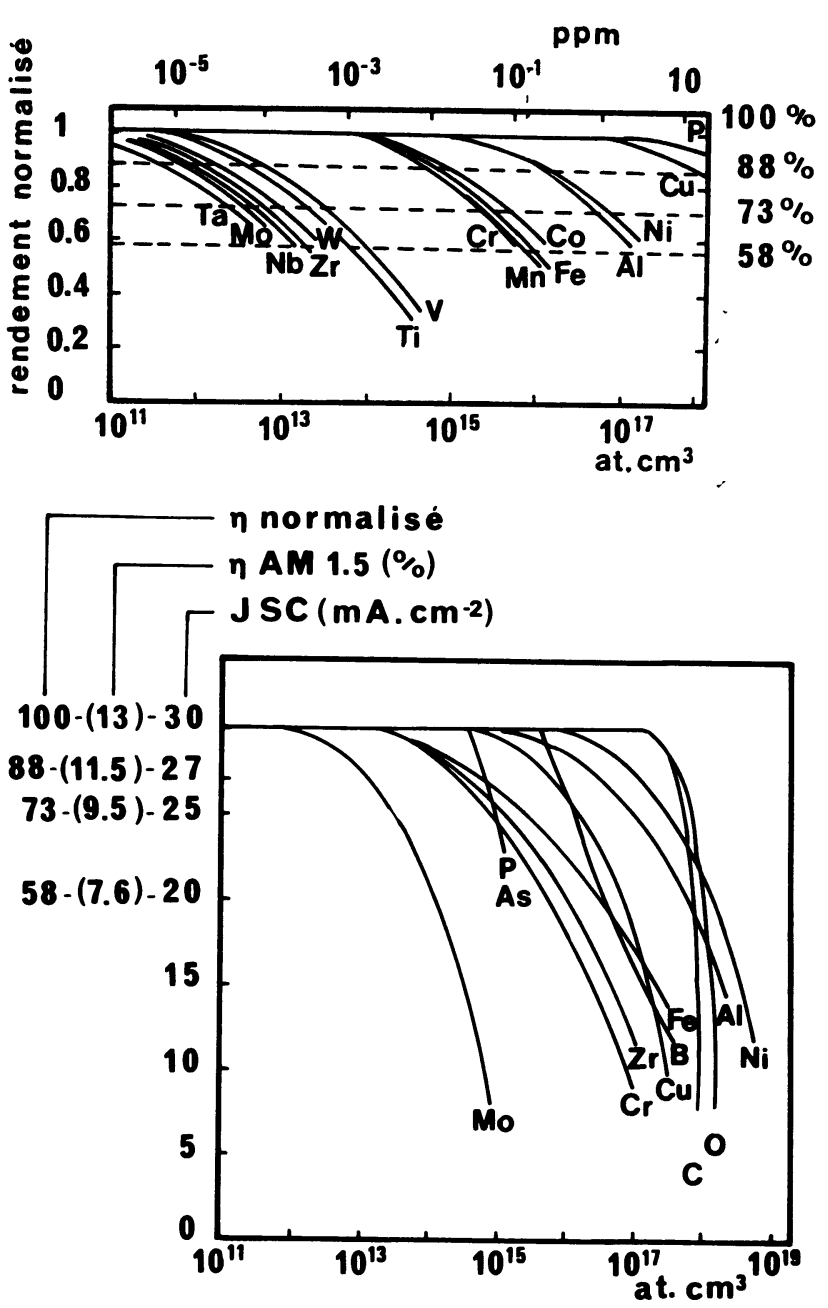

Fig. 14. - a) Action des impuretés sur les propriétés photovoltaïques $\left(\rho=2 \Omega \mathrm{cm}\right.$, cellules $\left.100 \mathrm{~cm}^{2}\right) \quad[10]$; b) Comparaison avec monocristal $\mathrm{CZ}$ type $\mathrm{p}$.

[a) Influence of impurities on the photovoltaic characteristics ( $\rho=2 \Omega \mathrm{cm}, 100 \mathrm{~cm}^{2}$ area ) [10] ; b) [p-type monocristal $\mathrm{CZ}$ ]. 
6.4 PASSivation Des DÉfauts « GetTERING ». Des études sur la passivation des défauts sont menées sur Polix dans plusieurs laboratoires. Ces études concernent la passivation par l'hydrogène, l'aluminium ou l'oxygène (effet «getter»).

$\mathrm{Au}$ stade actuel de connaissance du matériau et de réalisation des cellules, l'oxygène est une source de dégradation des performances. De nombreuses études visent à utiliser la présence de l'oxygène pour piéger les impuretés (effet "getter») tel qu'il est pratiqué de façon empirique pour certains dispositifs électroniques. L'élément de base pour expliquer les mécanismes mis en jeu est l'interface silicium-silice, zone où des accumulations d'impuretés ont été mises en évidence. L'étude du mécanisme de "gettering" nécessite l'utilisation de technique de microscopie électronique en transmission haute résolution et des méthodes d'analyse fine de surface.

Un exemple illustratif de l'influence de l'oxygène et des traitements thermiques résulte de la caractérisation photoélectrochimique du matériau.

Cette méthode est utilisée pour évaluer les performances photovoltaïques du matériau avant réalisation des cellules. En effet, lorsque la caractérisation est réalisée en polarisation cathodique sous éclairement, le courant mesuré est proportionnel au courant de court-circuit de la cellule. Les plaques sont caractérisées par référence à une plaque monocristalline.

Il s'est avéré que sur le matériau brut la validité de la méthode n'était pas assurée, il n'y a pas de différence sensible entre les plaques à bon rendement $(\simeq 11 \%)$ et celles à rendement médiocre $(\simeq 9 \%)$, les résultats sont tous alignés sur ceux de bonnes plaques. Par contre, si les plaques sont diffusées selon le procédé standard puis décapées pour éliminer la zone diffusée, la corrélation du courant obtenu par la méthode photoélectrochimique avec les performances des cellules est alors satisfaisante.

Un résultat identique a été obtenu par des mesures de conductance sur bicristaux, ces mesures ont en outre spécifié la sensibilité de la cristallographie des joints à ce traitement $[13,14]$.

Le traitement dégrade donc les performances de certains matériaux. Cette dégradation serait liée à la concentration en oxygène. Des analyses quantitatives vont être réalisées afin de corréler le niveau de dégradation et la teneur en oxygène. Pour utiliser l'effet «getter», il s'agit de mettre au point une méthode de piégeage des impuretés là où elles ne gêneront pas le dispositif de la cellule. La possibilité d'utiliser du silicium de qualité moindre contribue à l'intérêt des études des mécanismes mis en jeu. Il en est de même pour les méthodes de passivation des défauts $(\mathrm{H}, \mathrm{Al})$ qui sont d'autant plus efficaces que la cellule de départ présente un rendement faible.

\section{Conclusion.}

L'amélioration de la qualité du matériau qui s'est traduite par de très bonnes propriétés photovoltaïques et un coût de production compétitif est due aux caractérisations effectuées. Leurs corrélations avec les conditions d'élaboration ont permis l'adoption de solutions face aux problèmes posés par l'élaboration de lingots de grandes dimensions. L'association d'un encapsulant liquide qui a déterminé les conditions optimales de la nucléation à la solidification unidirectionnelle du bain, a conduit à l'obtention de lingots homogènes de $60 \mathrm{~kg}$.

La caractérisation du matériau a porté à la fois sur la morphologie des lingots, sur les défauts cristallographiques et sur les concentrations des différents éléments présents : défauts, carbone, oxygène, métaux, ... Il en est ressorti que les performances photovoltaïques des cellules solaires du type Polix dépendent davantage de la qualité cristalline que de l'état de pureté. Dans ce cas précis, l'amélioration a donc porté sur les paramètres thermiques qui contrôlent la solidification, et elle s'est concrétisée par de nombreux lingots qui présentent un rendement de conversion moyen de $11 \%$, pour atteindre des rendements supérieurs à $12,5 \%$ sur des cellules solaires standards.

Le point de fonctionnement actuel, durée du cycle voisine de vingt heures, coût des matières consommables limité autorise l'élaboration de lingots à des prix compétitifs si le rendement matière est supérieur à $50 \%$. Cette étape a été récemment franchie et sera dépassée prochainement par l'optimisation constante des paramètres thermiques et physiques qui interviennent lors de l'élaboration du lingot de silicium polycristallin et des cellules solaires.

L'utilisation de silicium de qualité moindre est liée à la mise en place de méthodes de caractérisation systématiques du matériau afin de déterminer des spécifications précises.

\section{Remerciements.}

Ce travail a bénéficié du soutien de l'Agence Française pour la Maîtrise de l'Energie. Nous tenons à remercier Mr J. Fally des Laboratoires de Marcoussis pour son étroite collaboration. 


\section{Bibliographie}

[1] Fally, J., Guenel, C., Proceeding of the International Conference of Photovoltaic Solar Energy, Cannes (1980) 598.

[2] Donon, J., Lauvray, H., Lay, P., Fally, J., Beaudereau, C., Proceeding of the IEEE, Orlando (1984) 296.

[3] Lauvray, H., Lay, P., Nouet, G., Donon, J., Proceeding of the Sixth Energy Conference Photovoltaic Solar, Londres (1985) (Ed. by Reidel, R., Publishing Company, Dordrecht) 1985, p. 901.

[4] Chermant, J. L., Coster, M., Lay, P., Nouet, G., Fourth European Symposium for Stereology, Göteborg (1985). Acta Stereol. 5 (1986) 299.

[5] Dash, W. C., J. Appl. Phys. 27 (1956) 1193.

[6] Sirtl, E., Adler, A., Z. Metallk. 52 (1961) 529.

[7] SECCO, D. F., J. Electrochem. Soc. 119 (1972) 948.
[8] SoPORI, B. L., J. Electrochem. Soc. 131 (1984) 667.

[9] Bary, A., Mercey, B., Poullain, G., Chermant, J. L., NoueT, G., ce volume.

BARY, A., Thèse de Doctorat, $3^{\text {e }}$ Cycle, Caen (1984).

[10] Fally, J., Guignot, D., Symposium on « Polymicro-crystalline and amorphous semiconductors », E-MRS, Strasbourg (1984) (Ed. by Pinard, P., Kalbitzer, S., Ed. de Physique, Paris) 1984, p. 199.

[11] Runyan, W. R., Silicon Semiconductor Technology (Ed. by McGraw Hill, New York) 1965.

[12] Bathey, B. R., Cretella, M. C., J. of Mater. Sci. 17 (1982) 3077.

[13] Poullain, G., Mercey, B., Nouet, G., J. Appl. Phys. 61 (1987) 1547.

[14] Poullain, G., Thèse de Doctorat, Paris (1985). 